\title{
Preparing for the COVID-19 Pandemic Response in a Country Emerging From an Ebola Epidemic: Assessment of Health Workers' Knowledge, Attitudes and Practices on Coronavirus (COVID-19) in Guinea
}

Ibrahima CAMARA ( ibcamara31@gmail.com )

Research article

Keywords: COVID-19, Assessment, Health Workers, Knowledge, Attitudes, Practices, and Guinea

Posted Date: May 15th, 2020

DOI: https://doi.org/10.21203/rs.3.rs-25099/v1

License: (c) (i) This work is licensed under a Creative Commons Attribution 4.0 International License. Read Full License 


\section{Abstract}

\section{Background:}

On January 30, 2020, the World Health Organization designated the outbreak as a Public Health Emergency of International Scope (USPPI). Underdeveloped countries, such as the Republic of Guinea, where the health system has been severely impacted by the Ebola virus epidemic between 2013 and 2014 , will therefore find it difficult to respond effectively to the predicted epidemic. The purpose of this study was to assess the knowledge, attitudes and practices of medical personnel on the prevention of Covid-2019.

\section{Methods:}

This was a cross-sectional study with an analytical aim carried out from 1st to 29th February 2020 among front-line health workers in the health structures of the city of Conakry. Data were collected using the Kobocollect application by administration of a standardized questionnaire. The knowledge and attitude score was categorized in 2: Good if $\geq$ at the average score and bad if $<50 \%$. Logistic regression models were conducted to identify factors associated with knowledge and attitude. Odds ratios (OR) with their confidence intervals were calculated.

\section{Results :}

548 health workers surveyed, the mean score for knowledge on the prevention of Covid-19 was 54.81 SD = 9.71, [95\% Cl: [ 53.9-55.6]. 70.6\% had a good knowledge of Covid-19. However, 99.6\% of the staff did not know the emergency number in case of a suspected case and about $50 \%$ did not know the incubation period of Covid-19.

Among the health personnel interviewed, $57.7 \%$ had a good attitude towards a suspected case of Covid19.

Although the majority $(57.5 \%)$ of the health workers had received training in infection prevention and control, infection prevention and control (IPC) practices were not routinely implemented, as the implementation rate was not even $80 \%$ for every action.

Variables associated with poor knowledge of Covid-19 prevention were sex, health facility, and staff grade. As for poor attitude, the associated variables were knowledge of Ebola cases reported in the facility and the rank of the health staff.

\section{Conclusion:}

This study underlines the difficulties of the appropriation of prevention and protection measures against Covid-19 by health personnel for whom the risk appears remote and limited to a transnational or foreign elite. 


\section{Background}

On the 9th of January 2020, following reports of a series of unexplained cases of Pneumonia, China announced that the city of Wuhan - capital of Hubei Province - was facing an outbreak linked to a new type of coronavirus, known as SARS-coV-2. The World Health Organization (WHO) qualified this epidemic on Thursday, January 30, 2020, as a Public Health Emergency of International Concern (PHEIC). Today, the epicentre of the epidemic has moved from China to Europe, demonstrating the great speed with which the epidemic is spreading through the flow of goods and people [1, 2]. 1,2] To date, 184 countries have reported cases of CoV-2-SARS infection. According to the World Health Organization's current assessment of the epidemic, 809,608 people have been infected, with 3,9033 deaths, representing a fatality rate of between 2 and $4.7 \%$ [3].

The known clinical manifestations of Covid-19 infection are similar to those of common influenza. The majority of patients complain of fever, cough, headache, runny nose, and in some cases dyspnea, muscle aches, $[1,4,5]$. The first Chinese patients had visited a local sea fish and wildlife market in the same province [6]. Human-to-human transmission is known to occur through airborne droplets that are sprayed directly onto people or deposited on soiled objects [7].

The reservoir of the virus is still unknown although coronaviruses are recognized as pathogens of animal origin mainly from camels, cattle, bats and rats $[8,9]$.

In fact, on the 28th of march 2020 there were 2831 confirmed cases, including 412 deaths in Africa. However, it should be noted that there is strong community transmission. These cases are distributed in 39 countries out of 52, including Guinea. On the 19th of March, the Ministry of Health of the Republic of Niger notified its first positive case of Covid-19. He was a 36-year-old Nigerien, a warehouseman for a land transport company who had travelled by road from Lomé to Abidjan to Accra, Ouagadougou and Niamey. The identification of a land-based spread of the epidemic prefigures a wider spread of cases, which are no longer limited to air travellers who are more easily identifiable and controllable.

In the Republic of Guinea, 31 cases were notified by the National Health Security Agency (NHSA) on the first of April 2020, including two cases with no epidemiological link and one case that was cured according to the national health security agency [11]. This meant the beginning of community transmission in the country, which was later confirmed (111 cases diagnosed on the 4th of April (ANSS). This situation came at a time when questions arose about the quality of the investigation of the second case (a Guinean national returned from a business trip to Italy), revealing that the patient presented herself in three health structures without mentioning her trip to Europe. In each case, a diagnosis of malaria would have been made before the patient was finally referred to a specialized centre in Conakry. About 1,000 contact cases for this patient are now being monitored and contained. This situation, which revealed the failure to identify at-risk cases and their systematic referral to referral centres, demonstrates the need for training and upgrading of health personnel on the COVID-19 pandemic. 
Despite the impact of the preparedness policies put in place on the continent over the past several years (reflected in particular in the capacity of national laboratories to carry out diagnosis), the Covid-19 epidemic highlights the weakness of coordination among countries in their response and the impact of budget cuts on health systems. Everywhere the epidemic reveals the weaknesses of health systems (limited capacity in intensive care units, lack of health personnel). Underdeveloped countries with known weak health systems will therefore find it difficult to respond effectively to the predicted epidemic. This is the case in the Republic of Guinea, where the already weak health system was severely impacted by the Ebola epidemic between 2013 and 2014 [12-14].

Part of the response to the outbreak of the epidemic involves raising public awareness and training health workers. Thus, to guide health authorities on the need for training and to direct the response towards targeted actions, we organized in February 2020, a few weeks before the notification of the first case of COVID-19, a survey among health personnel to assess knowledge, attitudes and practices to prevent coronavirus. The results of this study will help fill gaps in the training of front-line staff and the preparation of the health system for a possible increase in the number of cases.

The purpose of this study was to assess the knowledge, attitudes and practices of health personnel about COVID-19 and to identify their determinants.

\section{The objectives of this study were to:}

1. Describe the knowledge and attitudes of health personnel in the city of Conakry on COVID-19;

2. To identify the factors influencing the knowledge and attitudes of the health personnel on COVID-19.

\section{Methods}

\section{Study design:}

This was a cross-sectional analytical study conducted between first and 29th of February 2020 among frontline health personnel in the health structures of the city of Conakry (communal medical centres and the two national hospitals of Conakry).

\section{Framework of the study:}

The study was conducted in five communal medical centres (Coleah, Ratoma, le Flamboyant, Matam and Bernard KOUCHNER), three national hospitals (Donka and Ignace Deen and Sino-Guinean) and three health centres (Maciré, Dixinn and Madina). These hospitals are all located in the city of Conakry, the capital of the Republic of Guinea, which has an estimated population of 1,930,838 [15].

The majority of the health workforce is concentrated in this capital city and all specialized health services are also located here. This unequal distribution of personnel is responsible a significant influx of patients to these facilities, some of whom even come from the for interior of the country. 


\section{Study Participants :}

The study focused on front-line health workers in health facilities of Conakry. All health workers present on the day of the survey who gave their verbal consent were eligible for the survey. This is a face-to-face verbal interview with the respondent. All the answers were transferred to the tablets from and transferred to a server at the Infectious Disease Research and Training Center in Guinea.

We sampled for convenience in the selection of health services. This type of sampling was used to select those health services in which staff are considered convenient data sources for this study. Convenience sampling is a type of non-probability sampling in which individuals are sampled simply because they are "convenient" data sources for researchers [16].

The number of health personnel expected in the health facilities in Conakry was 900 . The respondent was asked about those present in the services and with a verbal agreement to participate in the study.

\section{Data Collection and Variables}

Data were collected by interviewers using a standardized questionnaire with 4 sections as follows:

1-socio-demographic characteristics such as age, gender, health structure, number of years of experience, academic degree, socio-professional category., 2-knowledge about the coronavirus, 3- attitudes to prevention and control of infection, 4- practices and information needs. The Kobocollect application was used to collect the data. The questionnaires were pre-tested for validation purposes. Imperfections in the questionnaire were corrected and the minimum time required to administer the questionnaire was estimated at 20 minutes.

Thirty (30) research assistants were mobilized to collect data in the eleven health structures. They were distributed according to the number of staff and the number of services included in the study.

In order to carry out this survey as quickly as possible because of the public health emergency it represented, we adapted the questionnaire used to assess health workers' knowledge of Ebola to Covid19 [17] and completed it using information provided by the Center for Disease Control and Prevention $(C D C)$ and WHO on the epidemic $[3,5]$.

\section{Knowledge Scoring :}

Knowledge and attitudes were assessed using structured questions on the virus, symptoms, care, prevention and response activities undertaken by the State. A total of 70 questions to assess knowledge and six (6) attitudinal questions were asked. Each correct answer was scored as 1, while the incorrect answer was scored as 0 . 
Good knowledge was defined as a score $\geq$ at the overall average and poor knowledge was defined as a score $<$ at the overall average. The same method was used to identify good and bad attitudes towards COVID-19.

To minimize bias, for each respondent the question was administered by a single interviewer and in an isolated office.

\section{Data Analysis:}

The data collected on kobo collect were extracted and cleaned before being analysed with Stata 14 software. Surveys with a lot of missing data were excluded from the final analysis. The basic characteristics are presented in proportion, mean and median as appropriate. Standard means and standard deviations and medians were used to describe the quantitative variables. A student or WilcoxonMann-Whitney t-test was used for comparison. The Chi2 or fisher exact test was used for the comparison of quality-avoidance variables.

Two logistic regression models were constructed to identify independent factors associated with poor knowledge and COVID-19's poor attitude to prevention.

In our analysis, we retained the variables whose $p$ was $\leq 20 \%$ in the bivariate regression analysis. The bottom-up stepwise method was used. The likehood ratio test was used to compare the successive model.

The final model has been validated by the Link test and the overall fit by the Hosmer-Lemeshow goodness-of-fit test. We also evaluated the discriminant power of the model with the ROC curve. A discriminant power was judged to be good if the ROC $\geq 80 \%$.

\section{Ethical Considerations:}

The study was approved by the Ethics and Scientific Committee of the Public Health department of the Faculty of Health Sciences and Technology of Gamal Abdel Nasser University, Conakry.

\section{Results:}

Between first and 26th of February 2020, we surveyed 548 frontline health workers in Conakry (capital of the Republic of Guinea) i.e. $60 \%$ of the expected sample (Fig. 1).

Nine health workers were excluded from the final analysis due to missing data because they had not completed the interview with the interviewers

More than half of the respondents were under the age of 30 with a median age of 29 years IQ (25-38). The majority of the agents interviewed were women (57.1\%) and had less than 5 years' experience in the 
field $(54.9 \%)$ and were nurses in $28 \%$ of cases. The majority of the participants were surveyed at Donka National Hospital $130 / 548$ or $23.7 \%$. Nurses were the most represented in the study (28.1\%). Most of the health staff reported that the service had not reported any cases of Ebola during the 2013-2016 epidemic) (Table 1). 
Table 1

Socio-demographic Characteristics of Health Personnel in Conakry, February 2020

\begin{tabular}{|c|c|c|}
\hline Variables & $N(548)$ & $\%$ \\
\hline \multicolumn{3}{|l|}{ Age (range) } \\
\hline Under 30 years old & 303 & 55.3 \\
\hline More than 30 years old & 245 & 44.7 \\
\hline Median (IQ) & 29 years IQ $(25-38)$ & \\
\hline \multicolumn{3}{|l|}{ Sex } \\
\hline Female & 313 & 57.1 \\
\hline Male & 235 & 42.9 \\
\hline \multicolumn{3}{|l|}{ Work experience in years } \\
\hline Less than 5 years old & 300 & 54.9 \\
\hline $5-10$ years & 142 & 25.8 \\
\hline More than 15 years old & 106 & 19.2 \\
\hline Average $(95 \% \mathrm{Cl})$ & 6 IC at $95 \%[5.5-6.1]$. & \\
\hline \multicolumn{3}{|l|}{ Structure surveyed } \\
\hline CMC Bernard Kouchner & 31 & 5.7 \\
\hline CMC Coleah & 47 & 8.6 \\
\hline CMC Flamboyant & 39 & 7.1 \\
\hline CMC Matam & 64 & 11.7 \\
\hline CMC Ratoma & 61 & 11.1 \\
\hline Dixinn Health Centre (Pharmaguinea) & 26 & 4.7 \\
\hline Maciré Health Centre (Dixinn) & 23 & 4.2 \\
\hline Madina Corniche Health Centre & 42 & 7.7 \\
\hline Donka National Hospital & 130 & 23.7 \\
\hline Ignace Deen National Hospital & 54 & 9.9 \\
\hline HN Sino-Guinean & 31 & 5.7 \\
\hline \multicolumn{3}{|l|}{ Grade of health personnel } \\
\hline Technical health worker & 50 & 9.1 \\
\hline
\end{tabular}




\begin{tabular}{|lll|}
\hline Variables & $\mathbf{N ~ ( 5 4 8 )}$ & $\%$ \\
\hline Biologist & 47 & 8.6 \\
\hline Students & 97 & 17.7 \\
\hline Nurse & 154 & 28.1 \\
\hline Internal & 79 & 14.4 \\
\hline Doctor & 95 & 17.3 \\
\hline Specialist physician & 19 & 3.5 \\
\hline Pharmacist & 7 & 1.3 \\
\hline Notified Ebola case in the structure & & \\
\hline Don't know. & 207 & 37,77 \\
\hline No & 230 & 41,97 \\
\hline Yes & 111 & 20,26 \\
\hline
\end{tabular}

\section{Knowledge And Attitudes Of Health Care Personnel About Covid-19}

The mean knowledge score on COVID-19 was $54.81+/-9.71$, (Table 2 ). When reporting on $100 \%$ of the scores obtained by each participant, the majority of respondents $(70.6 \%)$ had a score above $54.8 \% \mathrm{Cl}$ $95 \%$ [66.6-74.40], i.e. good knowledge of COVID-19.

Six (6) questions were asked to health personnel to assess their attitudes towards a suspicious case of COVID-19. Among the health workers interviewed, the mean attitude score was 62.3 ET $62.3+/-27.2$. On this basis, $57.7 \% 95 \% \mathrm{Cl}$ [5340 - 61.5] of the study participants had a good attitude towards a suspected case of COVID-19 (Table 2).

Almost all, 546/548 evening $99.6 \%$ of the interviewees did not know the emergency number in case of a suspected case and about 50\% did not know the incubation period of COVID-19.

Indeed, a large majority (53.8\%) of staff reported wanting to consult and prescribe medication for a patient with signs related to COVID-19.

There was a statistical association between gender, age, grade, health structure and level of knowledge. For attitude, the associated variables were age $(p=0.01)$, health structure $(p=0.01)$, health status $(p=$ $0.01)$ and knowledge $(p=0.01)$. 
Table 2

Comparison of socio-demographic characteristics of health workers in Conakry and their knowledge and attitudes on COVID-19, February 20202

\begin{tabular}{|c|c|c|c|c|c|c|}
\hline \multirow[t]{2}{*}{ Variables } & $\begin{array}{l}\text { Good } \\
\text { knowledge }\end{array}$ & $\begin{array}{l}\text { Poor } \\
\text { knowledge }\end{array}$ & $\mathbf{P}$ & $\begin{array}{l}\text { Good } \\
\text { Attitude }\end{array}$ & $\begin{array}{l}\text { Bad } \\
\text { Attitude }\end{array}$ & $\mathbf{P}$ \\
\hline & $\begin{array}{l}n=387 \\
(70.62 \%)\end{array}$ & $\begin{array}{l}n=161 \\
(29.38)\end{array}$ & & $\begin{array}{l}n=316 \\
(57.66 \%)\end{array}$ & $\begin{array}{l}n=232 \\
(42.34 \%)\end{array}$ & \\
\hline Work experience & & & 0.21 & & & 0.31 \\
\hline Less than 5 years old & $203(67.7)$ & $97(32.3)$ & & $166(55.3)$ & $134(44.7)$ & \\
\hline $5-10$ years & $106(75.2)$ & $35(24.8)$ & & $82(58.2)$ & $59(41.8)$ & \\
\hline More than 15 years old & $77(73.3)$ & $28(26.7)$ & & $67(63.8)$ & $38(36.2)$ & \\
\hline \multicolumn{7}{|l|}{ Median (IQ) } \\
\hline Age range & & & 0.005 & & & 0.01 \\
\hline Under 30 years old & $199(65.7)$ & $104(34.3)$ & & $161(53.1)$ & $142(46.9)$ & \\
\hline More than 30 years old & $188(76.7)$ & $57(23.3)$ & & $155(63.3)$ & $90(36.7)$ & \\
\hline \multicolumn{7}{|l|}{ Median (IQ) } \\
\hline Sex & & & $\begin{array}{l}\mathrm{P}< \\
0,001\end{array}$ & & & 0.66 \\
\hline Female & $198(63.3)$ & $115(36.7)$ & & $178(56.9)$ & $135(43.1)$ & \\
\hline Male & $189(80.4)$ & $46(19.6)$ & & $138(58.7)$ & $97(41.3)$ & \\
\hline Health structure surveyed & & & 0.008 & & & 0.01 \\
\hline CMC Bernard Kouchner & $19(61.3)$ & $12(38.7)$ & & $18(58.1)$ & $13(41.9)$ & \\
\hline CMC Coleah & $40(85.1)$ & $7(14.9)$ & & $35(74.5)$ & $12(25.5)$ & \\
\hline CMC Flamboyant & $27(69.2)$ & $12(30.8)$ & & $22(56.4)$ & $17(43.6)$ & \\
\hline CMC Matam & $33(51.6)$ & $31(48.4)$ & & $28(43.8)$ & $36(56.3)$ & \\
\hline CMC Ratoma & $43(70.5)$ & $18(29.5)$ & & $44(72.1)$ & $17(27.9)$ & \\
\hline $\begin{array}{l}\text { Dixinn Health Centre } \\
\text { (Pharmaguinea) }\end{array}$ & $15(57.7)$ & $11(42.3)$ & & $14(53.8)$ & $12(46.2)$ & \\
\hline $\begin{array}{l}\text { Maciré Health Centre } \\
\text { (Dixinn) }\end{array}$ & $18(78.3)$ & $5(21.7)$ & & 8 (34.8) & 15 (65.2) & \\
\hline $\begin{array}{l}\text { Madina Corniche Health } \\
\text { Centre }\end{array}$ & $30(71.4)$ & $12(28.6)$ & & $27(64.3)$ & 15 (35.7) & \\
\hline HN Donka & $97(74.6)$ & $33(25.4)$ & & 70 (53.8) & $60(46.2)$ & \\
\hline
\end{tabular}




\begin{tabular}{|c|c|c|c|c|c|c|}
\hline \multirow[t]{2}{*}{ Variables } & $\begin{array}{l}\text { Good } \\
\text { knowledge }\end{array}$ & $\begin{array}{l}\text { Poor } \\
\text { knowledge }\end{array}$ & $\mathbf{P}$ & $\begin{array}{l}\text { Good } \\
\text { Attitude }\end{array}$ & $\begin{array}{l}\text { Bad } \\
\text { Attitude }\end{array}$ & $\mathbf{P}$ \\
\hline & $\begin{array}{l}n=387 \\
(70.62 \%)\end{array}$ & $\begin{array}{l}n=161 \\
(29.38)\end{array}$ & & $\begin{array}{l}n=316 \\
(57.66 \%)\end{array}$ & $\begin{array}{l}n=232 \\
(42.34 \%)\end{array}$ & \\
\hline HN Ignace Deen & $39(72.2)$ & $15(27.8)$ & & $30(55.6)$ & $13(41.9)$ & \\
\hline HN Sino-Guinean & $26(83.9)$ & $5(16.1)$ & & $20(64.5)$ & $12(25.5)$ & \\
\hline Grade & & & $\begin{array}{l}P< \\
0,001\end{array}$ & & & 0.01 \\
\hline Technical health worker & $29(58)$ & $21(42)$ & & $20(40)$ & $30(60)$ & \\
\hline Biologist & $38(80)$ & $9(19.1)$ & & $30(63.8)$ & $17(36.2)$ & \\
\hline Students & $58(59)$ & $39(40.2)$ & & $50(51.5)$ & $47(48.5)$ & \\
\hline Nurse & $102(66)$ & $52(33.8)$ & & $93(60.4)$ & $61(39.6)$ & \\
\hline Internal & $59(74.7)$ & $20(25.3)$ & & $49(62)$ & $30(38)$ & \\
\hline Doctor & $80(84.2)$ & $15(15.8)$ & & $59(62.1)$ & $36(37.9)$ & \\
\hline Specialist physician & $18(94.7)$ & $1(5.3)$ & & $14(73.7)$ & $5(26.3)$ & \\
\hline Pharmacist & $3(42.9)$ & $4(57.1)$ & & $1(14.3)$ & $6(85.7)$ & \\
\hline
\end{tabular}

\section{$\mathrm{PCl}$ practices and information sources}

Participants' infection prevention and control practices were assessed using a Likert scale (Fig. 1), Study participants reported that they always disinfected surfaces, utensils and work equipment $(69.9 \%)$ and then washed their hands after a medical procedure (76.6\%). They also reported that they always wore boots $(26.3 \%)$, gloves $(63 \%)$ and gowns $(48.7 \%)$ during a medical procedure. Soap and hydro-alcoholic solutions were the most commonly used by staff, with proportions of $55.7 \%$ and $42.5 \%$ respectively (Table 3).

It should be noted, however, that infection prevention and control (ICP) was not systematically applied by all staff. Thus, preventive gestures were not systematically applied (the rate of application did not even reach $80 \%$ for each gesture). (Fig. 2) 
Table 3

Prevention behavior of COVID-19 among health personnel in Conakry, February 2020

\begin{tabular}{|c|c|c|}
\hline Variables & $N(548)$ & $\%$ \\
\hline \multicolumn{3}{|c|}{$\begin{array}{l}\text { What product do you have it on } \\
\text { your hands with? }\end{array}$} \\
\hline With water & 10 & 1.8 \\
\hline With soap and water & 305 & 55.7 \\
\hline With a disinfectant & 233 & 42.5 \\
\hline \multicolumn{3}{|c|}{$\begin{array}{l}\text { How often do you have physical contact } \\
\text { (Handshake, etc.) per day }\end{array}$} \\
\hline Between 50 and 100 & 49 & 8.9 \\
\hline Minus 50 & 353 & 64.4 \\
\hline Don't know. & 132 & 24.1 \\
\hline More than 100 & 14 & 2.6 \\
\hline
\end{tabular}

\section{Sources of information on COVID-19 for health care personnel-19 (Fig. 2)}

The media was the main source of information about COVID-19 for health care workers (81\%), both online and offline. The professional and family network was the main source of information for staff, with $65 \%$ and $35 \%$ respectively. (Fig. 3)

Most health personnel (57.5\%) had already received training in infection prevention and control and wanted more information on COVID-19 (98\%) . 
Table 4

Need for information and training on

COVID-19 for health personnel in

Conakry, February 20202

\begin{tabular}{|c|c|c|}
\hline Variables & $N(N=548)$. & $\%$ \\
\hline \multicolumn{3}{|c|}{ Have you received PCI training? } \\
\hline No & 233 & 42.5 \\
\hline Yes & 315 & 57.5 \\
\hline \multicolumn{3}{|c|}{$\begin{array}{l}\text { You think you need more } \\
\text { any information on Coronavirus? }\end{array}$} \\
\hline No & 11 & 2 \\
\hline Yes & 537 & 98 \\
\hline
\end{tabular}

\section{Factors associated with poor knowledge of COVID among health care personnel-19 ( Table 5 )}

After a logistic regression, the variables associated with poor knowledge of COVID are gender, the structure where the respondent worked, the fact of having trained in ICP in the past, and rank.

Indeed, women, compared to men, are $2.4695 \% \mathrm{Cl}$ [1.62-3.74] times more likely to have a poor knowledge of COVID-19, all other things being equal with $p=0.001$.

Healthcare workers who have had ICP training in the past compared to those who have not, are $40 \%$ less likely to have poor knowledge of COVID-19 (OR $=0.61 \mathrm{Cl}[0.40-0.89], \mathrm{P}=0.01$. Pharmacists compared to health technical officers (HTAs) are more likely to have poor knowledge of COVID-19. ( $P=9.8395 \% \mathrm{Cl}$ [1.09-38.44]).

For the independent determinants of poor attitude, the associated variables are: Knowledge of Ebola cases reported in the department, health facility and grade of health worker. 
Table 5

Factors associated with the poor knowledge of health personnel in Conakry about covid-19, February 20

\begin{tabular}{lllllll} 
Variables & $\begin{array}{l}\text { Raw } \\
\text { gold }\end{array}$ & $P>\| z \mid$ & {$[95 \% \mathrm{Cl}]$} & $\begin{array}{l}\text { OR } \\
\text { adjust }\end{array}$ & $P>\| z \mid$ & {$[95 \% \mathrm{C}]$} \\
\hline Sex & & & & & & \\
Male & & & & 1 Ref & & \\
Female & 2.38 & 0.001 & $\begin{array}{l}{[1.60-} \\
3.54]\end{array}$ & 2,46 & 0,001 & {$[1.62-$} \\
& & & & & $3.74]$
\end{tabular}

\section{Structures}

CMC Bernard Kouchner

1 Réf

1 ref

CMC Coleah

0.27

$0.02 \quad[0.09-$

$0.81]$

0.35

0.01

[0.15-

$0.81]$

CMC Flamboyant

0.45

$\begin{array}{ll}0.40 \quad[0.26- \\ & 1.89]\end{array}$

0.61

0.38

[0.20-

CMC Matam

1.48

$0.37 \quad[0.63-$
$5 / 56]$

1.99

0.02

[1.133.46]

CMC Ratoma

0.66

$\begin{array}{ll}0.37 \quad[0.26- \\ & 1.64]\end{array}$

0.95

0.93

$[0.35-$

2.59]

Dixinn Health Centre

(Pharmaguinea)

Maciré Health Centre (Dixinn)

Madina Corniche Health Centre

HN Donka

HN Ignace Deen

HN Sino-Guinean

1.16

0.78

$[0.40-$

3.35]

0.43

$\begin{array}{ll}0.12 \quad[0.12- \\ & 1.14]\end{array}$

0.63

0.33

$[0.23-$

$1.66]$

$0.14 \quad[0.23-$

$1.27]$

0.60

0.29

[0.23-

0.30

0.05

[0.09-
$1.01]$

0.36

0.55

0.59

0.62

0.37

0.76

[0.373.89]

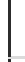

IPC training in the past

\begin{tabular}{lllllll} 
No & 1 Ref & \multicolumn{7}{c}{ 1ref } & & \\
Yes & 0.53 & 0.001 & $\begin{array}{l}{[0.36-} \\
0.77]\end{array}$ & 0.61 & 0.01 & {$[0.40-$} \\
& & & & & $0.89]$
\end{tabular}

Grade of health personnel

Technical health worker

1 Ref

1 Ref 


\begin{tabular}{|c|c|c|c|c|c|c|}
\hline Variables & $\begin{array}{l}\text { Raw } \\
\text { gold }\end{array}$ & $P>\| z \mid$ & {$[95 \% \mathrm{Cl}]$} & $\begin{array}{l}\text { OR } \\
\text { adjust }\end{array}$ & $P>\| z \mid$ & [95\% Cl] \\
\hline Biologist & 0.32 & 0.01 & $\begin{array}{l}{[0.13-} \\
0.81]\end{array}$ & 0.30 & 0.017 & $\begin{array}{l}{[0.11-} \\
0.80]\end{array}$ \\
\hline Students & 0.92 & 0.83 & $\begin{array}{l}{[0.46-} \\
1.86]\end{array}$ & 0.96 & 0.93 & $\begin{array}{l}{[0.45-} \\
2.07]\end{array}$ \\
\hline Nurse & 0.70 & 0.29 & $\begin{array}{l}{[0.36-} \\
1.35]\end{array}$ & 0.67 & 0.28 & $\begin{array}{l}{[0.32-} \\
1.39]\end{array}$ \\
\hline Internal & 0.46 & 0.05 & $\begin{array}{l}{[0.21-} \\
0.99]\end{array}$ & 0.61 & 0.26 & $\begin{array}{l}{[0.26-} \\
1.44]\end{array}$ \\
\hline Doctor & 0.25 & 0.001 & $\begin{array}{l}{[0.11-} \\
0.56]\end{array}$ & 0.39 & 0.03 & $\begin{array}{l}{[0.16-} \\
0.96]\end{array}$ \\
\hline Specialist physician & 0.07 & 0.016 & $\begin{array}{l}{[0.09-} \\
0.62]\end{array}$ & 0.11 & 0.04 & $\begin{array}{l}{[0.01-} \\
0.96]\end{array}$ \\
\hline Pharmacist & 1.84 & 0.45 & $\begin{array}{l}{[0.37-} \\
9.10]\end{array}$ & 9.83 & 0.03 & $\begin{array}{l}{[1.09-} \\
38.44]\end{array}$ \\
\hline
\end{tabular}


Table 6

Determinants of the poor attitude of health personnel in Conakry in the face of a suspected case of COVID-19, February 2020

\begin{tabular}{|c|c|c|c|c|c|c|}
\hline Variables & Raw gold & $P>\| z \mid$ & [95\% Cl] & OR adjust & $P>\| z \mid$ & {$[95 \% \mathrm{Cl}]$} \\
\hline \multicolumn{7}{|l|}{$\begin{array}{l}\text { Knowledge of Ebola cases } \\
\text { notified in }\end{array}$} \\
\hline \multicolumn{7}{|l|}{ The service } \\
\hline No & $\begin{array}{l}1 \\
\text { Reference }\end{array}$ & & & & & \\
\hline Yes & 1.42 & 0.13 & $\begin{array}{l}{[0.89-} \\
2.27]\end{array}$ & 1.40 & 0.22 & $\begin{array}{l}{[0.81-} \\
2.41]\end{array}$ \\
\hline Don't know. & 1.89 & 0.001 & $\begin{array}{l}{[1.28-} \\
2.78]\end{array}$ & 1.57 & 0.01 & $\begin{array}{l}{[1.09-} \\
2.25]\end{array}$ \\
\hline \multicolumn{7}{|l|}{ Structures } \\
\hline CMC Bernard Kouchner & $\begin{array}{l}1 \\
\text { Reference }\end{array}$ & & & $\begin{array}{l}1 \\
\text { reference }\end{array}$ & & \\
\hline CMC Coleah & 0.47 & 0.13 & $\begin{array}{l}{[0.18-} \\
1.25]\end{array}$ & 0.44 & 0.02 & $\begin{array}{l}{[0.22-} \\
0.88]\end{array}$ \\
\hline CMC Flamboyant & 1.06 & 0.89 & $\begin{array}{l}{[0.14-} \\
2.77]\end{array}$ & 1.36 & 0.54 & $\begin{array}{l}{[0.49-} \\
3.76]\end{array}$ \\
\hline CMC Matam & 1.78 & 0.19 & $\begin{array}{l}{[0.74-} \\
4.23]\end{array}$ & 1.85 & 0.20 & $\begin{array}{l}{[0.72-} \\
4.46]\end{array}$ \\
\hline CMC Ratoma & 0.53 & 0.17 & $\begin{array}{l}{[0.21-} \\
1.32]\end{array}$ & 0.51 & 0.02 & $\begin{array}{l}{[0.27-} \\
1.90]\end{array}$ \\
\hline $\begin{array}{l}\text { Dixinn Health Centre } \\
\text { (Pharmaguinea) }\end{array}$ & 1.18 & 0.74 & $\begin{array}{l}{[0.41-} \\
3.39]\end{array}$ & 0.55 & 0.02 & $\begin{array}{l}{[0.27-} \\
0.92]\end{array}$ \\
\hline Maciré Health Centre (Dixinn) & 2.59 & 0.09 & $\begin{array}{l}{[0.85-} \\
7.95]\end{array}$ & 2.43 & 0.13 & $\begin{array}{l}{[0.75-} \\
7.87]\end{array}$ \\
\hline Madina Corniche Health Centre & 0.76 & 0.58 & $\begin{array}{l}{[0.29-} \\
1.99]\end{array}$ & 0.87 & 0.79 & $\begin{array}{l}{[0.31-} \\
2.39]\end{array}$ \\
\hline HN Donka & 1.18 & 0.67 & $\begin{array}{l}{[0.53-} \\
2.62]\end{array}$ & 1.30 & 0.50 & $\begin{array}{l}{[0.55-} \\
3.26]\end{array}$ \\
\hline HN Ignace Deen & 1.10 & 0.82 & $\begin{array}{l}{[0.45-} \\
2.70]\end{array}$ & 1.30 & 0.59 & $\begin{array}{l}{[0.49-} \\
3.43]\end{array}$ \\
\hline HN Sino-Guinean & 0.76 & 0.60 & $\begin{array}{l}{[0.27-} \\
2.12]\end{array}$ & 0.96 & 0.94 & $\begin{array}{l}{[0.30-} \\
3.01]\end{array}$ \\
\hline
\end{tabular}




\begin{tabular}{|c|c|c|c|c|c|c|}
\hline Variables & Raw gold & $P>|| z \mid$ & [95\% Cl] & OR adjust & $P>\| z \mid$ & {$[95 \% \mathrm{Cl}]$} \\
\hline Technical health worker & $\begin{array}{l}1 \\
\text { Reference }\end{array}$ & & & $\begin{array}{l}1 \\
\text { Reference }\end{array}$ & & \\
\hline Biologist & 0.37 & 0.02 & $\begin{array}{l}{[0.16-} \\
0.85]\end{array}$ & 0.32 & 0.32 & $\begin{array}{l}{[0.13-} \\
0.78]\end{array}$ \\
\hline Students & 0.62 & 1.18 & $\begin{array}{l}{[0.31-} \\
1.25]\end{array}$ & 0.45 & 0.45 & $\begin{array}{l}{[0.20-} \\
0.97]\end{array}$ \\
\hline Nurse & 0.43 & 0.01 & $\begin{array}{l}{[0.22-} \\
0.83]\end{array}$ & 0.36 & 0.36 & $\begin{array}{l}{[0.18-} \\
0.74]\end{array}$ \\
\hline Internal & 0.40 & 0.016 & $\begin{array}{l}{[0.19-} \\
0.84]\end{array}$ & 0.32 & 0.32 & $\begin{array}{l}{[0.14-} \\
0.71]\end{array}$ \\
\hline Doctor & 0.40 & 0.012 & $\begin{array}{l}{[0.20-} \\
0.82]\end{array}$ & 0.44 & 0.44 & $\begin{array}{l}{[0.20-} \\
0.92]\end{array}$ \\
\hline Specialist physician & 0.23 & 0.016 & $\begin{array}{l}{[0.07-} \\
0.76]\end{array}$ & 0.23 & 0.23 & $\begin{array}{l}{[0.06-} \\
0.78]\end{array}$ \\
\hline Pharmacist & 4 & 0.21 & $\begin{array}{l}{[0.44-} \\
35.78]\end{array}$ & 9.83 & 0.03 & $\begin{array}{l}{[1.15-} \\
83.62]\end{array}$ \\
\hline
\end{tabular}


Table 7

Comparison of the knowledge and attitudes of health workers in Conakry regarding a suspected case of COVID-19 and their IPC practices - 19, February 20202

\begin{tabular}{|c|c|c|c|c|c|c|}
\hline \multirow[t]{2}{*}{ Variables } & $\begin{array}{l}\text { Good } \\
\text { knowledge }\end{array}$ & $\begin{array}{l}\text { Poor } \\
\text { knowledge }\end{array}$ & $\mathbf{P}$ & $\begin{array}{l}\text { Good } \\
\text { attitude }\end{array}$ & $\begin{array}{l}\text { Bad } \\
\text { attitude }\end{array}$ & $\mathbf{P}$ \\
\hline & $\begin{array}{l}n=387 \\
(70.62)\end{array}$ & $\begin{array}{l}n=161 \\
(29.38)\end{array}$ & & $\begin{array}{l}n=316 \\
(57.66 \%)\end{array}$ & $\begin{array}{l}n=232 \\
(42.34 \%)\end{array}$ & \\
\hline $\begin{array}{l}\text { In the last three months, have } \\
\text { you changed your work habits } \\
\text { by }\end{array}$ & & & 0.002 & & & 0.000 \\
\hline No & $69(59)$ & $48(41)$ & & $\begin{array}{l}37 \\
(31.6)\end{array}$ & $\begin{array}{l}80 \\
(68.4)\end{array}$ & \\
\hline Yes & $318(73.8)$ & $113(26.2)$ & & $\begin{array}{l}279 \\
(64.7)\end{array}$ & $\begin{array}{l}152 \\
(35.3)\end{array}$ & \\
\hline $\begin{array}{l}\text { How often do you have } \\
\text { physical contact (handshake, } \\
\text { etc.)? }\end{array}$ & & & 0.02 & & & 0.02 \\
\hline \multicolumn{7}{|l|}{ in the middle of the day } \\
\hline Between 50 and 100 & $40(81.6)$ & $9(18.4)$ & & $\begin{array}{l}27 \\
(55.1)\end{array}$ & $\begin{array}{l}22 \\
(44.9)\end{array}$ & \\
\hline Minus 50 & $257(72.8)$ & $96(27.2)$ & & $\begin{array}{l}218 \\
(61.8)\end{array}$ & $\begin{array}{l}135 \\
(38.2)\end{array}$ & \\
\hline Don't know. & $80(60.6)$ & $52(39.4)$ & & $62(47)$ & $70(53)$ & \\
\hline More than 100 & $10(71.4)$ & $4(28.6)$ & & $\begin{array}{l}9 \\
(64.29)\end{array}$ & $\begin{array}{l}5 \\
(35.71)\end{array}$ & \\
\hline $\mathrm{PCl}$ training in the past & & & 0.001 & & & 0.007 \\
\hline No & 147 (63.1) & 86 (36.9) & & $\begin{array}{l}119 \\
(51.1)\end{array}$ & $\begin{array}{l}114 \\
(48.9)\end{array}$ & \\
\hline Yes & $240(76.2)$ & $75(23.8)$ & & $\begin{array}{l}197 \\
(62.5)\end{array}$ & $\begin{array}{l}118 \\
(37.5)\end{array}$ & \\
\hline Use of gowns: & & & 0.09 & & & 0.23 \\
\hline Never & $49(66.2)$ & $25(33.8)$ & & $\begin{array}{l}38 \\
(51.4)\end{array}$ & $\begin{array}{l}36 \\
(48.6)\end{array}$ & \\
\hline Sometimes & $28(56)$ & $22(44)$ & & $30(60)$ & $20(40)$ & \\
\hline Rarely & $29(69)$ & $13(31)$ & & $\begin{array}{l}23 \\
(54.8)\end{array}$ & $\begin{array}{l}19 \\
(45.2)\end{array}$ & \\
\hline Often & $82(71.3)$ & $33(28.7)$ & & $\begin{array}{l}59 \\
(51.3)\end{array}$ & $\begin{array}{l}56 \\
(48.7)\end{array}$ & \\
\hline
\end{tabular}




\begin{tabular}{|c|c|c|c|c|c|c|}
\hline \multirow[t]{2}{*}{ Variables } & $\begin{array}{l}\text { Good } \\
\text { knowledge }\end{array}$ & $\begin{array}{l}\text { Poor } \\
\text { knowledge }\end{array}$ & $\mathbf{P}$ & $\begin{array}{l}\text { Good } \\
\text { attitude }\end{array}$ & $\begin{array}{l}\text { Bad } \\
\text { attitude }\end{array}$ & $\mathbf{P}$ \\
\hline & $\begin{array}{l}n=387 \\
(70.62)\end{array}$ & $\begin{array}{l}n=161 \\
(29.38)\end{array}$ & & $\begin{array}{l}n=316 \\
(57.66 \%)\end{array}$ & $\begin{array}{l}n=232 \\
(42.34 \%)\end{array}$ & \\
\hline Always & $199(74.5)$ & $68(25.5)$ & & $\begin{array}{l}166 \\
(62.2)\end{array}$ & $\begin{array}{l}101 \\
(37.8)\end{array}$ & \\
\hline Wearing gloves : & & & 0.89 & & & 0.4 \\
\hline Never & $10(62.5)$ & $6(37.5)$ & & $7(43.8)$ & $9(56.3)$ & \\
\hline Sometimes & $29(74.4)$ & $10(25.6)$ & & $\begin{array}{l}19 \\
(48.7)\end{array}$ & $\begin{array}{l}20 \\
(51.3)\end{array}$ & \\
\hline Rarely & $13(65)$ & $7(35)$ & & $10(50)$ & $10(50)$ & \\
\hline Often & $90(70.3)$ & $38(29.7)$ & & $73(57)$ & $55(43)$ & \\
\hline Always & $245(71)$ & $100(29)$ & & $207(60)$ & $138(40)$ & \\
\hline Wearing the face mask: & & & 0.3 & & & 0.18 \\
\hline Never & $69(63.9)$ & $39(36.1)$ & & $\begin{array}{l}58 \\
(53.7)\end{array}$ & $\begin{array}{l}50 \\
(46.3)\end{array}$ & \\
\hline Sometimes & $100(71.9)$ & $39(28.1)$ & & $\begin{array}{l}79 \\
(56.8)\end{array}$ & $\begin{array}{l}60 \\
(43.2)\end{array}$ & \\
\hline Rarely & $44(73.3)$ & $16(26.7)$ & & $\begin{array}{l}28 \\
(46.7)\end{array}$ & $\begin{array}{l}32 \\
(53.3)\end{array}$ & \\
\hline Often & $67(68.4)$ & $31(31.6)$ & & $\begin{array}{l}62 \\
(63.3)\end{array}$ & $\begin{array}{l}36 \\
(36.7)\end{array}$ & \\
\hline Always & $107(74.8)$ & $36(25.2)$ & & $\begin{array}{l}89 \\
(62.2)\end{array}$ & $\begin{array}{l}54 \\
(37.8)\end{array}$ & \\
\hline Eye Protection : & & & 0.12 & & & 0.013 \\
\hline Never & $123(66.8)$ & $61(33.2)$ & & $\begin{array}{l}90 \\
(48.9)\end{array}$ & $\begin{array}{l}94 \\
(51.1)\end{array}$ & \\
\hline Sometimes & $78(69.6)$ & $34(30.4)$ & & $\begin{array}{l}69 \\
(61.6)\end{array}$ & $\begin{array}{l}43 \\
(38.4)\end{array}$ & \\
\hline Rarely & $69(67.6)$ & $33(32.4)$ & & $\begin{array}{l}56 \\
(54.9)\end{array}$ & $\begin{array}{l}46 \\
(45.1)\end{array}$ & \\
\hline Often & 37 (71.2) & $15(28.8)$ & & $\begin{array}{l}36 \\
(69.2)\end{array}$ & $\begin{array}{l}16 \\
(30.8)\end{array}$ & \\
\hline Always & $80(81.6)$ & $18(18.4)$ & & $\begin{array}{l}65 \\
(66.3)\end{array}$ & $\begin{array}{l}33 \\
(33.7)\end{array}$ & \\
\hline Wear boots/cover boots : & & & 0.81 & & & 0.001 \\
\hline
\end{tabular}




\begin{tabular}{|c|c|c|c|c|c|c|}
\hline \multirow[t]{2}{*}{ Variables } & $\begin{array}{l}\text { Good } \\
\text { knowledge }\end{array}$ & $\begin{array}{l}\text { Poor } \\
\text { knowledge }\end{array}$ & \multirow[t]{2}{*}{$\mathbf{P}$} & $\begin{array}{l}\text { Good } \\
\text { attitude }\end{array}$ & $\begin{array}{l}\text { Bad } \\
\text { attitude }\end{array}$ & \multirow[t]{2}{*}{$\mathbf{P}$} \\
\hline & $\begin{array}{l}\mathrm{n}=387 \\
(70.62)\end{array}$ & $\begin{array}{l}n=161 \\
(29.38)\end{array}$ & & $\begin{array}{l}n=316 \\
(57.66 \%)\end{array}$ & $\begin{array}{l}n=232 \\
(42.34 \%)\end{array}$ & \\
\hline Never & 105 (66.9) & $52(33.1)$ & & $80(51)$ & $77(49)$ & \\
\hline Sometimes & $74(71.8)$ & $29(28.2)$ & & $70(68)$ & $33(32)$ & \\
\hline Rarely & $53(71.6)$ & $21(28.4)$ & & $\begin{array}{l}33 \\
(44.6)\end{array}$ & $\begin{array}{l}41 \\
(55.4)\end{array}$ & \\
\hline Often & $50(71.4)$ & $20(28.6)$ & & $\begin{array}{l}50 \\
(71.4)\end{array}$ & $\begin{array}{l}20 \\
(28.6)\end{array}$ & \\
\hline Always & $105(72.9)$ & $39(27.1)$ & & $\begin{array}{l}83 \\
(57.6)\end{array}$ & $\begin{array}{l}61 \\
(42.4)\end{array}$ & \\
\hline \multirow{3}{*}{$\begin{array}{l}\text { Which product do you use } \\
\text { you wash your hands? }\end{array}$} & & & 0.78 & & & \\
\hline & & & & & & \\
\hline & & & & & & 0.48 \\
\hline With water & $8(80)$ & $2(20)$ & & $6(60)$ & $4(40)$ & \\
\hline With soap and water & $216(70.8)$ & $89(29.2)$ & & $\begin{array}{l}169 \\
(55.4)\end{array}$ & $\begin{array}{l}136 \\
(44.6)\end{array}$ & \\
\hline \multirow[t]{2}{*}{ With a disinfectant } & $163(70)$ & $70(30)$ & & $\begin{array}{l}141 \\
(60.5)\end{array}$ & $\begin{array}{l}92 \\
(39.5)\end{array}$ & \\
\hline & & & 0.09 & & & \\
\hline $\begin{array}{l}\text { How often do you wash } \\
\text { and Disinfect the surfaces } \\
\text { and utensils }\end{array}$ & & & & & & 0.001 \\
\hline Never & $6(60)$ & $4(40)$ & & $2(20)$ & $8(80)$ & \\
\hline Sometimes & $15(51.7)$ & $14(48.3)$ & & $\begin{array}{l}11 \\
(37.9)\end{array}$ & $\begin{array}{l}18 \\
(62.1)\end{array}$ & \\
\hline Rarely & $14(82.4)$ & $3(17.6)$ & & $6(35.3)$ & $\begin{array}{l}11 \\
(64.7)\end{array}$ & \\
\hline Often & $97(68.3)$ & $45(31.7)$ & & $\begin{array}{l}72 \\
(50.7)\end{array}$ & $\begin{array}{l}70 \\
(49.3)\end{array}$ & \\
\hline Always & 255 (72.9) & $95(27.1)$ & & $\begin{array}{l}225 \\
(64.3)\end{array}$ & $\begin{array}{l}125 \\
(35.7)\end{array}$ & \\
\hline
\end{tabular}

Discussion 
This study assessed the attitudes, knowledge and practices of health personnel in Conakry on COVID-19 and identify factors associated with poor knowledge and attitude of these practitioners. $70.62 \%$ of the personnel have a good knowledge of COVID-19 and $57.66 \%$ have a good attitude of disease prevention. The majority of the knowledge acquired on the issue was from the media and internet (81\%). The health system did not put in place anything to help the staff to become familiar with the fight because $99.60 \%$ did not know the emergency number and did not have a management algorithm (53. $\left.80 \%^{\circ}\right)$.

Although it was a cross-sectional survey with the possibility of information bias due to the fact that the questionnaires were not self-administered. Some staff would have had the correct answer if they had received the question beforehand. This study has the advantage of giving us an overview of the knowledge and attitudes of health staff regarding COVID-.19 in the pandemic preparedness situation.

Since the beginning of this pandemic, all efforts have been focused on management and actions to break the chain of transmission of COVID-19. The first articles published on the subject focused on the overall understanding and characteristics of the virus $[4,7,18]$. To our knowledge, no studies have yet explored the issue of the knowledge, attitudes and practices of front-line health care workers regarding COVID-19. To fill in the gaps on the issue and to guide decision-makers on the strategy for involving health care personnel, we conducted a knowledge, attitudes and practices survey among 548 health care personnel on COVID-19.

This study shows us that there are still some gains from the previous Ebola epidemic in terms of knowledge, attitudes and infection prevention and control practices.Indeed, it was observed that the majority of health workers have a good knowledge of Covid- 19 (70\% of the sample). However, the overwhelming majority of health workers, all statuses and structures combined (99.6\%) were unaware of the existence of a toll-free number to alert on the presence of a suspected case of coronavirus and only $42.3 \%$ knew the incubation period of the virus. In a similar study of Ebola in Guinea, $95.2 \%$ of the health workers were knowledgeable and could identify an Ebola suspect [19]. Although this is not the same context, nor the same pathogen, there is an effort on the part of health workers to learn about public health threats. This is reflected in the sources of information consulted: $81 \%$ of them obtained their information from the media (Internet, radio). The same source was used by $85 \%$ of the surveys in Sierra Leone to obtain information on Ebola [20].

1.

$57.1 \%$ faced with a patient with signs of COVID-19 said they would consider it but $37 \%$ would not. What seems even more worrisome is that $41.2 \%$ said they would prescribe a drug according to symptomatology or diagnosis, which raises the question of what happens to patients left to their own devices to pursue their quest for care (with a risk of spreading the virus). Also if $81.8 \%$ answered that they would wear PPE (Personal Protective Equipment) to consult them, it remains to be seen what they mean by this when in reality, all that is needed is a mask, gloves and protective glasses. This proportion of staff using personal protective equipment is almost equal to the proportion reported in Nigeria for assessing the PPE of health workers in the Ebola context [17]. 
$60.9 \%$ assured that in the event of a case with symptoms of Covid-19 they would put it in isolation and $62.6 \%$ announced that they would call the dedicated toll-free number. However, these responses clearly contained a significant desirability bias, since all the agents admitted that they were unaware of the existence of the toll-free number that would allow them to do so.

Systematic hand washing (76.6\% of respondents) and glove wearing $(63 \%)$ seemed to be relatively practiced but remain too weak to ensure effective protection for all agents and patients. The wearing of masks $(26.1 \%)$ is largely insufficient, as is the wearing of gowns $(48.7 \%)$. Concerning the literature on Haemorrhagic Fevers, the literature shows that the practice of handwashing varies according to whether health workers are practicing in a declared epidemic context or in an alert context. The practice was observed in the first case, around 80 to $85 \%$ in Sierra Leone and Nigeria $[17,20]$, but was very low in the second, as in Iran during the Crimean-Congo fever alert [21]. This issue is crucial for the implementation of infection prevention and control measures among health workers in the context of the COVID epidemic [19] where the feeling of health actors seems to be that if the epidemic is present, it is confined to a limited fringe of the population (elites and foreigners).

The importance of exercise conditions is clear in the question about the product used to wash hands. Only $42.5 \%$ had a hydroalcoholic gel and $55.7 \%$ used only soap and water.

$81.6 \%$ of health workers reported shaking less than 50 hands a day, which is already too much. It remains to be seen how many avoided all contact.

$42.5 \%$ of health workers have not received ICP training, and the vast majority of them (98\%) would like to receive more information on the Coronavirus. It would also be advisable to identify, through the observation of practices, which types of actors are mainly exposed due to their contact with patients in order to prioritize their training (we can think of actors developing frequent physical contact with patients: care assistants, nurses, but also wardens or reception agents, etc.) but also how the application of ICP impacts on the quality of care produced (in particular by the absence of palpation-type contact).

The agents in the health centres and National Hospitals, a large proportion of whom have less than 5 years of seniority (Health Centre: $47 \%$, HN $38 \%$ ) compared to the CMCs $(15 \%)$ had on average a better knowledge of the coronavirus (Health Centre and National Hospital 41.9\%, CMC 16.3). This could be explained by the fact that the issue of zoonoses and ICP is now part of the training curriculum for health workers (Faculty of Health Sciences and Techniques and Nursing School), in which they have participated. This therefore calls for priority to be given to upgrading the skills of health professionals with more than five years' seniority.

There is no strong correlation between level of knowledge of Covid-19 and expression of intention to advise isolation in case of symptoms, systematic mobilization of barrier gestures. This invites us to go beyond the cognitive question of knowledge of the risk to question both its perceptions, in particular the conviction of the actors to be concerned by the risk of the Coronavirus and the factors which do not come under the purely biomedical register which influences it (risk scales, rumours...), This is not only the 
political context which gives rise to concern, putting the question of viral risk in the background), but also the conditions for appropriating the knowledge acquired in ordinary practice (conditions of practice, presence of protective equipment or disinfectant products, influx of patients, leadership role of the medical hierarchy in the establishment). Particular attention should be paid to the impact of discourse on the extraneity of viral risk on the management of national patients. Until now, prevention measures have focused on airports and the figure of the virus carrier has been embodied by the Asian or European traveller. This figure has not been contradicted by the first proven cases. However, as we enter a phase of local transmission of the virus, it is extremely important to alert health workers to the presence of the virus on national territory and to the fact that it is not limited to a section of the population of non-African origin.

We have identified the independent determinants of health personnel's knowledge and attitudes about COVID-19.

For poor knowledge, it is the female sex, being a pharmacist, having benefited from training in ICP in the past and working in the communal medical centre of Matam, in fact, women are 2.46 at risk of having poor knowledge of COVID-19 compared to men $p=0.001$. This result could be explained by the gendered distribution of roles in the Guinean health system. The majority of men occupy positions of responsibility, which allows them to have easier access to information at the central level. On the other hand, they occupy a greater number of administrative positions that allow them to search for information, whereas women, who are mostly confined to care functions, have fewer opportunities. In the context of the Ebola fever epidemic on the African continent, the lack of time that women could devote to information seeking and their high exposure to risk due to their care functions has been put forward to explain their high lethality [22]. With regard to the pharmacy profession ( $O R=9.83 \mathrm{Cl}[1.09-38.44], p=0.03)$, it is possible that pharmacists working in health facilities or pharmacies may suffer from a lack of access to training and workshops on infection prevention and control compared to health care workers. Staff who have received ICP training in the past, the medical profession and biologists are likely to be knowledgeable about COVID.19

This may be directly associated with the effects of the Ebola epidemic from 2013 to 2016, during which much ICP training was organized for health workers.

Although we covered all public structures in the capital, this study has certain limitations: first of all, private structures were not included, nor were informal spaces for the production of care or the dispensing of medication products. In addition, assessing the level of knowledge about a new virus such as Covid-19, for which knowledge is evolving daily, may seem a challenge. In spite of these limitations, this study is of definite interest at a time when Guinea, along with the African continent, is organizing its response to the emergence of a new epidemic.

\section{Conclusion:}

The epidemic of COVID-19 disease is an "extraordinary" pandemic because of its global scale and the speed of its spread. Despite the collective trauma it is causing worldwide, it is important to recognize the contexts in which it is occurring. While in all countries medical personnel remain at the heart of the response mechanism, their level of knowledge and experience of the epidemic is context specific. If we 
observe the legacy of the previous Ebola epidemic among certain Guinean health workers (in terms of knowledge and practices), significant gaps remain and are the subject of a demand for training on the part of the actors. Our study has also highlighted the difficulties of the appropriation of prevention and protection measures against Covid-19 by health workers for whom the risk appears remote and limited to a transnational or foreign elite.

It would be advisable to associate to this CAP survey an observation of long-term practices allowing in situ observation of actors' practices in order to avoid desirability bias (an observation every day for a week allows actors to "forget" the presence of the observer). This would make it possible to work on the distinction between the official norm expressed in the discourse, and the practical rationalities relating to the conditions of practice, professional cultures, and the nature of the interaction between the health worker and the patient (which itself often depends as much on prior inter-knowledge as on the social status of the patient, for example).

\section{Abbreviations}

CMC : Communal medical center

CDC :Centers for Disease Control and Prevention

COVID : coronavirus disease

SARS: Severe Acute Respiratory Syndrome

NHSA: National Health Security Agency

$\mathrm{NH}:$ National hospital

Cl: Confidence interval

\section{Declarations}

\section{Authors' contributions}

IC : Conception de l'étude, mise en œuvre, analyse des données et écriture de l'article ;

AT : aparticiper à la conception, la validation des données et la relecture de l'article

AC, MSS : Ont participé à la conception, la validation des données et la relecture de l'article ;

FLM :A participation à la discussion, la validation des données et la correction de l'article et la traduction de l'articles en Anglais ;

BST : Participation à la conception de l'étude, à la supervision de l'enquête, la correction de l'article ; 


\section{Authors' Informations}

- Ibrahima CAMARA, ibcamara31@gmail.com

- Abdoulaye TOURE, toure@insp-guinee.org

- Alioune CAMARA, aliounec@gmail.com

- Frédéric LeMarcistoure@insp-guinee.org

- Salifou Tassone BANGOURA,talassoneb@gmail.com

- Mamamdou Saliou SOW, smsaliou@gmail.com

- Alpha Kabinet KEITA, alpha-kabinet.keita@cerfig.org

\section{Declaration of competing interest}

The authors declare no conflicts of interest.

\section{Remerciements:}

Nous tenons à remercier l'École Nationale Supérieure de Lyon, France pour le financement de l'étude et le centre de recherche et de formation en infectiologie pour avoir servit de cadre à la formation et au suivi de l'étude sur le terrain.

We would like to thank the École Nationale Supérieure de Lyon, France for funding the study and centre de recherche et de formation eninfectiologie de Guinée for providing the framework for the training and follow-up of the field study.

\section{Availability of data and materials}

The data are available from the corresponding author, it may be available after an express request

\section{Consent to publication:}

Not applicable

\section{Funding}

This project was co-funded by the authors and École Normale Supérieure de Lyon, France. 
The authors financed the costs of the field investigation, the publication costs are borne by École Normale Supérieure de Lyon, France, which also has a teacher who participated in the work.

\section{References}

1. Chen N, Zhou M, Dong X, Qu J, Gong F, Han Y, et al. Epidemiological and clinical characteristics of 99 cases of 2019 novel coronavirus pneumonia in Wuhan, China: a descriptive study. The Lancet 2020 Jan 30

2. Coronavirus disease 2019 [Internet]. [cited 2020 Mar 21]. Available from: https://www.who.int/emergencies/diseases/novel-coronavirus-2019

3. Novel Coronavirus (2019-nCoV) situation reports [Internet]. [cited 2020 Feb 6]. Available from: https://www.who.int/emergencies/diseases/novel-coronavirus-2019/situation-reports

4. Huang C, Wang Y, Li X, Ren L, Zhao J, Hu Y, et al. Clinical features of patients infected with 2019 novel coronavirus in Wuhan, China. The Lancet 2020 Jan 24

5. Transmission of Novel Coronavirus (2019-nCoV) | CDC2020 [cited 2020 Feb 6]. Available from: https://www.cdc.gov/coronavirus/2019-ncov/about/transmission.html

6. Lu H, Stratton CW, Tang Y-W. Outbreak of Pneumonia of Unknown Etiology in Wuhan China: the Mystery and the Miracle. J Med Virol 2020 Feb 6

7. Ghinai I, McPherson TD, Hunter JC, Kirking HL, Christiansen D, Joshi K, et al. First known person-toperson transmission of severe acute respiratory syndrome coronavirus 2 (SARS-CoV-2) in the USA. The Lancet 2020 Mar 13

8. CDC. Middle East Respiratory Syndrome (MERS) Centers for Disease Control and Prevention. 2019 [cited 2020 Feb 6]. Available from: https://www.cdc.gov/coronavirus/mers/index.html

9. SARS Severe acute respiratory syndrome | SARS-CoV disease I CDC 2019 [cited 2020 Feb 6]. Available from: https://www.cdc.gov/sars/index.html

10. 20200328-sitrep-68-covid-19.pdf. [cited 2020 Mar 29]. Available from: https://www.who.int/docs/default-source/coronaviruse/situation-reports/20200328-sitrep-68-covid19.pdf

11. (10) Agence Nationale de Sécurité Sanitaire-ANSS - Home [Internet]. cited 2020 Mar 29]. Available from:

https://www.facebook.com/Sanitaire.net/photos/rpp.194940584289708/882245148892578/? type=3heater

12. Elston JWT, Cartwright C, Ndumbi P, Wright J. The health impact of the 2014-15 Ebola outbreak. Public Health. 2017 Feb; 143:60-70.

13. Delamou A, Ayadi AME, Sidibe S, Delvaux T, Camara BS, Sandouno SD, et al. Effect of Ebola virus disease on maternal and child health services in Guinea: a retrospective observational cohort study. Lancet Glob Health. 2017;5(4):e448-57. 
14. Leno NN, Delamou A, Koita Y, Diallo TS, Kaba A, Delvaux T, et al. Ebola virus disease outbreak in Guinea: what effects on prevention of mother-to-child transmission of HIV services? Reprod Health. 2018 Apr 10;15(1):60.

15. Investigation reports - RGPH 2014 - stat-guinee.org [Internet]. cited 2020 Mar 29]. Available from: http://www.stat-guinee.org/index.php/publications-ins/rapports-d-enquetes/category/113-rgph-2014

16. Convenience Sampling. In: Encyclopedia of Survey Research Methods 2455 Teller Road, Thousand Oaks California 91320 United States of America: Sage Publications, Inc.; 2008

17. Iliyasu G, Ogoina D, Otu AA, Dayyab FM, Ebenso B, Otokpa D, et al. A Multi-Site Knowledge Attitude and Practice Survey of Ebola Virus Disease in Nigeria. PLoS ONE 2015 Aug 28

18. Chan JF-W, Yuan S, Kok K-H, To KK-W, Chu H, Yang J, et al. A familial cluster of pneumonia associated with the 2019 novel coronavirus indicating person-to-person transmission: a study of a family cluster. The Lancet 2020 Jan 24

19. Sidibé S, Camara BS, Delamou A, Touré A, Bouedouno P, Samake AT, et al. [Knowledge, attitudes and practices of healthcare providers on suspected Ebola cases in Guinea]. Rev Epidemiol Sante Publique. 2018 Nov;66(6):369-74.

20. Ebola-Virus-Disease-National-KAP-Study-Final-Report_-final.pdf [Internet]. [cited 2020 Mar 26]. Available from: https://reliefweb.int/sites/reliefweb.int/files/resources/Ebola-Virus-Disease-NationalKAP-Study-Final-Report_-final.pdf

21. Rahnavardi M, Rajaeinejad M, Pourmalek F, Mardani M, Holakouie-Naieni K, Dowlatshahi S. Knowledge and attitude toward Crimean-Congo haemorrhagic fever in occupationally at-risk Iranian healthcare workers. J Hosp Infect. 2008 May 1;69(1):77-85.

22. WHO | Ebola virus disease - Democratic Republic of Congo. WHO. World Health Organization; [cited 2020 Mar 26]. Available from: http://www.who.int/csr/don/07-february-2019-ebola-drc/fr/

\section{Figures}

900 expected

health personnel

343 were absent on the

days of the investigation

557health

personnel

548 included in

the final analysis

9 excluded because of missing data. 
Figure 1

Flow flowdiagram of the inclusion of participants in the

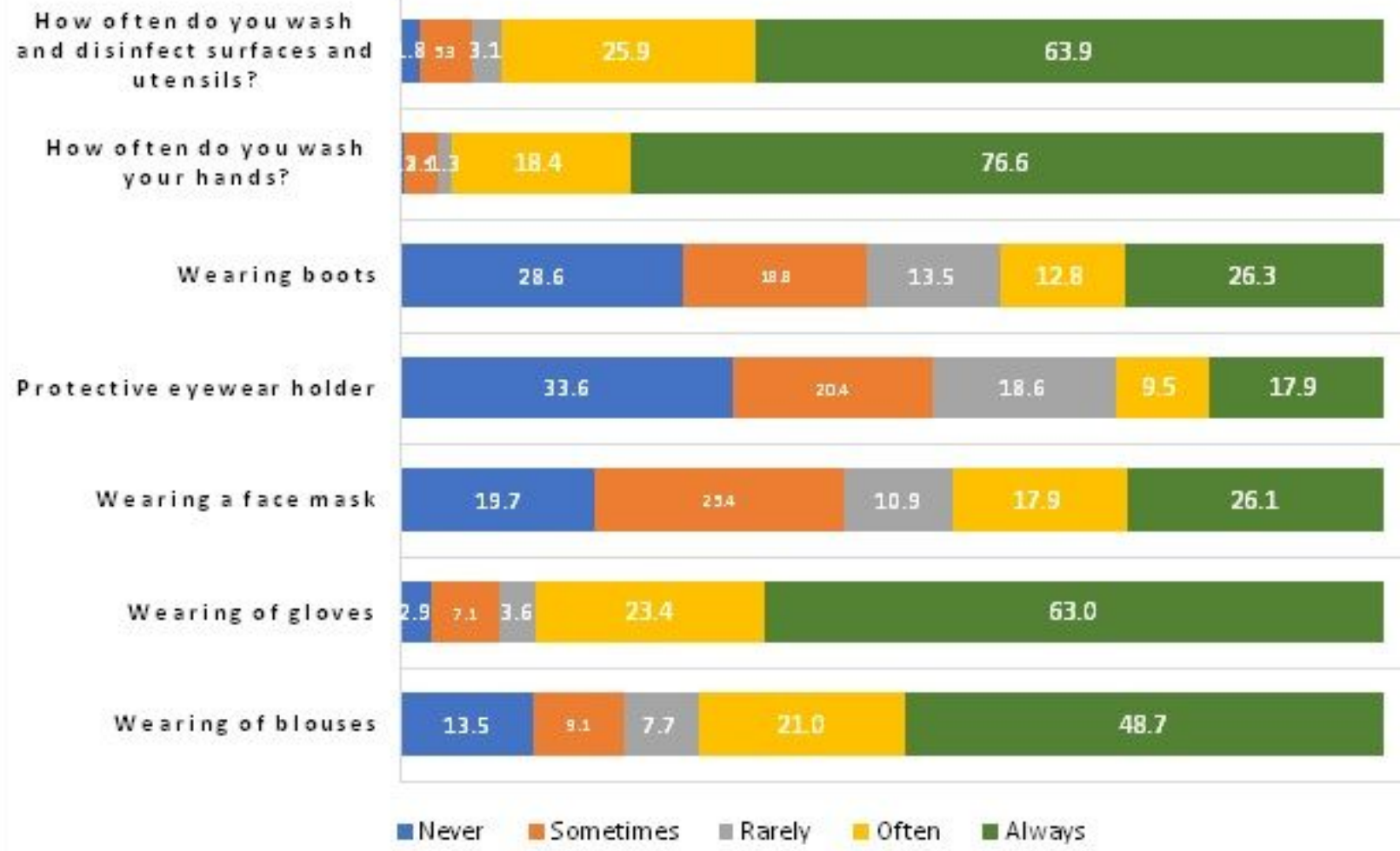

Figure 2

ICP practices of health personnel in Conakry, February 2020 ICP: Infection Control Prevention

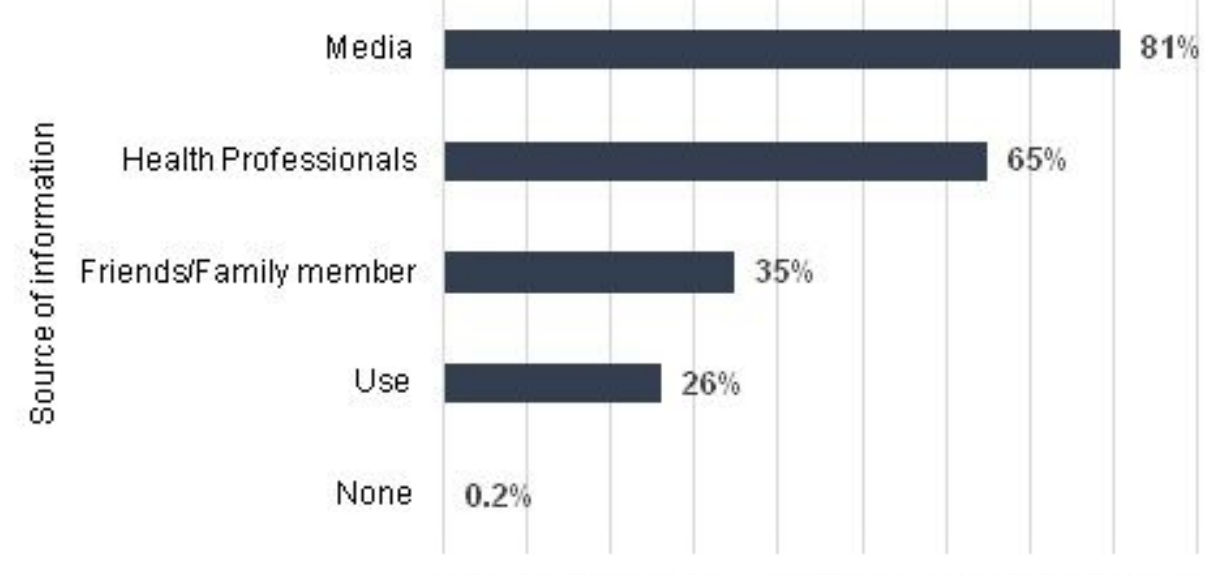

$0.0 \% 10.0 \% 20.0 \% 30.0 \% 40.0 \% 50.0 \% 60.0 \% 70.0 \% 80.0 \% 90.0 \%$

Percentage

\section{Figure 3}


Sources of informatio for Conakry health professionals on COVID-16, February 2020

\section{Supplementary Files}

This is a list of supplementary files associated with this preprint. Click to download.

- Authorsscontribution.docx 